\title{
Mejoramiento de la técnica ATPM para reducción de interferencia en sistemas de banda ancha por línea de potencia en residencias
}

\section{Improvement of the ATPM technique for interference reduction in systems of broadband by power line in residences}

\section{José Roberto Cárdenas Castiblanco}

Ingeniero Electrónico, Magister en Teleinformática, Doctor en Ingeniería. Profesor asociado de la Maestría en Ciencias de la Información y las Comunicaciones de la Universidad Distrital Francisco José de Caldas. Bogotá, Colombia. Contacto:rcardenas@udistrital.edu.co

Fecha de recepción: 30 de noviembre de 2012

Clasificación del artículo: investigación

Fecha de aceptación: 23 de noviembre de 2013

Financiamiento: Universidad Nacional de Colombia

Palabras clave: mitigación de la interferencia, técnica ATPM, variación de funciones de transferencia.

Key words: ATPM Technique, mitigation of the interference, variation of transfer functions.

\section{RESUMEN}

La interferencia electromagnética producida por los sistemas de comunicación de banda ancha por línea de potencia (en inglés, Broadband over Power Lines [BPL]) en redes residenciales es un aspecto técnico que no está totalmente solucionado. Este artículo presenta una técnica en la cual la longitud y la carga de los ramales de la red, cuya topología es de bus, son modificadas para variar la función de transferencia del canal entre un transmisor y un receptor, con el objeto de disminuir la densidad espectral de potencia (DEP) del transmisor. El algoritmo que desarrolla esta técnica hace uso de tres principios teóricos encontrados: la ganancia por distancia, la construcción discreta de la capacidad de canal y direccionalidad de los nodos de transmisión y recepción. Se estudió una red típica y, como resultado, combinando esta técnica con la técnica conocida como Adaptive Transmit Power Management (ATPM) se logró una reducción de $26.4 \mathrm{~dB}$ en la densidad espectral de potencia.

\section{ABSTRACT}

The electromagnetic interference produced by the In-House Broadband over Power Line (BPL) 


\section{investigación}

communication systems is a technical aspect that is not completely settled. This article presents a technique in which the length and load of the branches of the network, whose topology is bus, are modified to vary the transfer function of the channel between a transmitter and a receiver in order to reducing the Power Spectral Density (PSD) of the transmitter. The algorithm that develops this technique makes use of three theo- retical principles found: the gain by distance, the discrete construction of the channel capacity and directionality of the nodes of transmission and reception. A typical network was studied and as a result, combining this technique with the technique known as Adaptive Transmit Power Management (ATPM), there was a reduction of 26.4 $\mathrm{dB}$ in the power spectral density.

\section{INTRODUCCIÓN}

Estudios han mostrado que una importante fuente de interferencia electromagnética que está emergiendo en la banda de frecuencias de HF (3-30 $\mathrm{MHz}$ ) es producida por sistemas de para comunicaciones en banda ancha que usan las líneas de distribución de energía eléctrica (Stott, 2006). Se ha observado que esta interferencia sobrepasa el límite especificado por cuerpos nacionales como la Federal Communications Commission (FCC) (Federal Communications Commission, 2009) y las recomendaciones de la International Telecommunication Union (ITU) (ITU-R SM Series, 2011).

En diciembre de 2010, el Institute of Electrical and Electronics Engineers (IEEE) publicó el estándar 1901 (IEEE Communications Society, 2010) (cuya banda de operación es 1,8-30 MHz), el cual oficializó la tecnología que usa la transmisión de datos de banda ancha por línea de potencia (en inglés, Broadband Over Power Line [BPL]). En dicho estándar se especificó una densidad espectral de potencia (DEP) de transmisión de $-55 \mathrm{dBm} / \mathrm{Hz}$, valor que está lejano del valor estimado por Chende $-72 \mathrm{dBm} / \mathrm{Hz}$ (Cheng, 2003), el cual sería necesario para cumplir con lo especificado por la FCC para efectos de compatibilidad electromagnética. La máscara espectral de transmisión especificada por IEEE atenúa la DEP en las frecuencias usadas por los radioaficionados mediante un sistema de ranuras.
$*$



Figura 1. Máscara espectral de transmisión propuesta por IEEE 1901y límites recomendados

Fuente: IEEE Communications Society (2010).

ITU-R también se ha pronunciado en relación con la protección de las comunicaciones aeronáuticas (ITU-R SM Series, 2011) (sección A.2.6), y ha recomendado que los sistemas PLT (denominación europea para BPL) operando con una DEP de -55 $\mathrm{dBm} / \mathrm{Hz}$ deberían reducir la DEP en $48 \mathrm{~dB}$. En la figura 1 se muestra el actual estado de las recomendaciones de DEP para redes BPL.

De acuerdo con ITU-R el nivel de DEP recomendado de $-103 \mathrm{dBm} / \mathrm{Hz}$ se obtendría con una combinación de técnicas, para así poder reducir la DEP de transmisión en 18 dB usando control dinámico de potencia de transmisión (en inglés, Adaptive Transmit Power Management,-ATPM-) y en $30 \mathrm{~dB}$ usando técnicas de ranuras. De acuerdo con esto la técnica ATPM logra menores resultados si 
se compara con la técnica de ranuras. Este trabajo mejora la técnica ATPM, obteniendo mejoras de hasta $26,4 \mathrm{~dB}$ en la DEP de transmisión, haciéndola así equiparable con el rendimiento de la técnica de ranuras. Esta mejora se logró mediante un algoritmo que propone un aumento en la magnitud de la función de transferencia $(|\mathrm{H}(\mathrm{f})|)$ para el caso del canal de distribución eléctrica residencial.

\section{ESTADO DEL ARTE EN TÉCNICAS DE REDUCCIÓN DE INTERFERENCIA DE SISTEMAS BPL RESIDENCIALES}

Las corrientes de modo común generadas a lo largo de los cables de una línea de potencia y sus campos magnéticos asociados (causantes de la interferencia) son principalmente debidas a asimetrías eléctricas. De acuerdo con Favre et al. (2009), las corrientes de modo común pueden ser reducidas con el uso en los tomacorrientes de unos "insertos" y así disminuir el campo magnético radiado entre 6 y $10 \mathrm{~dB}$. Sin embargo, las pruebas realizadas se hacen en espacios pequeños y preocupa el hecho de aumentar en su operación la impedancia hacia tierra de protección.

Otra técnica se basa en colocar una interferencia destructiva intencional, inyectando una señal auxiliar seleccionada en el circuito Tierra-Neutro (Vukicevic et al., 2007). Sin embargo, es posible que no se pueda lograr una alineación entre las componentes del campo emitido y el destructivo, lo que puede resultar en que algunas componentes de la emisión sean incrementadas. Los autores argumentan que en promedio se logra una reducción de 10 a $20 \mathrm{~dB}$ en todas las componentes del campo en el área de interés. En pruebas de laboratorio, Pang et al. (2010) aseguran haber mejorado esta técnica inyectando una señal entre fase y tierra, y otra entre tierra y neutro.

Por otra parte, las conclusiones de la tesis doctoral de Mekuria (2008) señalan que es posible transmitir pulsos ultra wide band (UWB) en el intervalo nano sobre canales PLC y, con ello, se puede lograr una reducción de las interferencias del orden de $15 \mathrm{~dB}$ en frecuencias por debajo de $30 \mathrm{MHz}$. Esta propuesta llevaría a replantear totalmente la actual tecnología.

En un enfoque teórico, Chaaban et al. (2009) analizan una antena de hilo y representan el campo radiado en función de la reducción de la longitud y el radio del conductor de la antena, para demostrar que la potencia radiada es mínima para diferentes valores de longitud. A partir de este resultado se pueden escoger valores de frecuencia óptimos para limitar la potencia electromagnética radiada y, así, reducirla a alrededor de $7.5 \mathrm{~dB}$.

ITU-R (ITU-R SM Series, 2011), por su parte, solamente considera dos técnicas para reducción de la interferencia: ranuras dinámicas y ATPM. Existen dos versiones en la técnica de ranuras. En la primera las ranuras son estáticas, como se muestra el figura 1. La segunda técnica denominada de ranuras dinámicas (Weling, 2011) se basa en la detección de estaciones de radiodifusión y de acuerdo con ITU-R la reducción en la DEP es de $30 \mathrm{~dB}$. A continuación se trata la técnica ATPM.

\section{ADAPTIVE TRANSMIT POWER MANAGEMENT (ATPM)}

ATPM hace uso de la habilidad que tienen implementada los actuales módems BPL en el Control Automático de Ganancia (CAG) para amplificar la señal en el lado receptor. ATPM propone que, en vez de transmitir con un nivel de transmisión constante, tiene sentido reducir el nivel de transmisión y dejar que el CAG amplifique la señal recibida. Esto quiere decir que el modem debe detectar pérdidas de transmisión y fijar la potencia por transmitir al mínimo nivel requerido para lograr una transferencia de datos a una velocidad dada. Conviene aclarar que actualmente en general los módems BPL no tienen implementado ATPM. Schwager (2010) propone que usando 


\section{investigación}
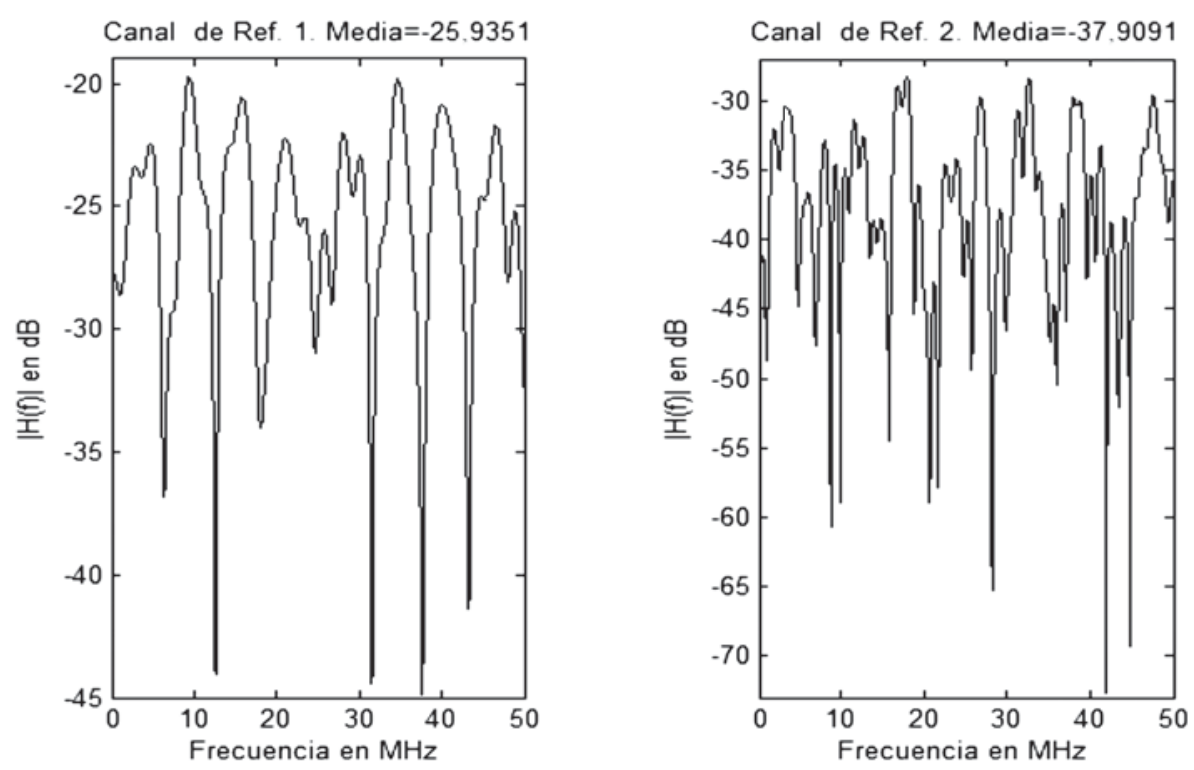

Figura 2. $|H(f)|$ de canales de referencia para BPL

Fuente: Datos suministrados por Babic et al. (2005).

ATPM se puede hacer la reducción de potencia en el transmisor mediante la ecuación (1).

\section{Reducción de potencia $(\mathrm{dB})=30 \mathrm{~dB}$}

+Atenuación mínima de canal (dB)

Es importante tratar en este punto el tipo de canal que cubre este artículo. Para ello es valioso el aporte de Babic et al. (2005), el cual consiste en la definición de cuatro canales de referencia para canales de banda ancha por línea de potencia. El presente estudio toma los dos primeros, los cuales corresponden a canales de redes de distribución eléctrica residenciales, cuyas magnitudes de función de transferencia se muestran en la figura 2 y las cuales se convierten en los límites sobre los que se trabajará. Cabe aclarar que la figura 2 se obtiene gracias a que Babic et al. proporcionaron los detalles de la respuesta al impulso de cada canal.

Como ejemplo se considera el canal de referencia 1 (gráfica de la izquierda en figura 2). En este caso la mínima atenuación del canal es -19,7056
dB. Al aplicar la ecuación (1) se obtiene una reducción de potencia de transmisión de 10,3 dB. Se observa que el cálculo de $18 \mathrm{~dB}$ de ITU-R con respecto a reducción de potencia con ATPM podría ser un poco optimista. Sin embargo surge la pregunta: ¿podría existir alguna técnica que permitiera obtener picos menores de $|\mathrm{H}(\mathrm{f})|$, para así lograr una mayor reducción de potencia? En lo que sigue se intentará responder esta pregunta.

\section{METODOLOGÍA}

Para encontrar la respuesta fue necesario seguir algunos pasos con diferentes diseños metodológicos, que a continuación se describen.

\section{Uso del método analítico}

A simple vista, para examinar el comportamiento de una red de transmisión de energía para uso con BPL sería suficiente con plantear las ecuaciones de una red en forma de bus compuesta por líneas 
de transmisión. La aproximación tradicional en este caso se basa en el método analítico, el cual algunas veces en la literatura se referencia como método bottom-up, donde se conocen los parámetros eléctricos de las líneas de transmisión, las cargas, sus longitudes y la estructura de la red. Sin embargo en el caso BPL residencial la tierra de protección introduce grandes variaciones en la $|\mathrm{H}(\mathrm{f})|$ del canal residencial, haciendo que su modelación no sea sencilla. No obstante, se encontraron algunas soluciones que permitieron usar el método analítico.

\section{Parámetros eléctricos para las líneas de transmisión de canales residenciales}

Bostoen y Van de Wiel (2003) hicieron un estudio con el cable de distribución de 4x95 $\mathrm{mm}^{2} \mathrm{y}$ los cables de conexión de 4x10 mm² y 4x25 mm². Todos estos cables se componen de cuatro conductores: un cable para neutro y tres conductores para cada fase. Sin embargo, estos cables se aproximan como líneas monofásicas al unir dos de los cuatro conductores (neutro y una fase) o al unir los conductores para las tres fases. Por tanto, se definen tres modos de conexión, 1-2, 1-3, y $1-(2+3+4)$.

Para estos modos de conexión, Bostoen y Van de Weil encontraron los parámetros eléctricos por unidad de longitud, los cuales dependen de la frecuencia y de los factores $r_{1}, l_{1}, l_{2}, g_{1}, c_{1}$, (ecuación (5)).

$$
\begin{gathered}
r=r_{1} \sqrt{f}, l=l_{1}+\frac{l_{2}}{\sqrt{f}} \\
g=g_{1} f \\
c=c_{1}
\end{gathered}
$$

Estos factores son listados por Bostoen y Van de Weil (2003) y cuando se utilizan en el cálculo de magnitudes de funciones de transferencia se observa un error promedio de $+/-1.13 \mathrm{~dB}$.

Por su parte, Barmada et al. (2009) también proporcionan los parámetros por unidad de longitud para una línea de transmisión residencial, pero se encuentra que estos parámetros tienen valores muy cercanos con la línea $4 \times 10 \mathrm{~mm}^{2}$ modo 1-3 de Bostoen y Van de Weil.

Herramienta de cálculo de las magnitudes de funciones de transferencia de canales en una red residencial

Algunas de las siguientes consideraciones han sido inspiradas en comentarios hechos por Tucci en Computational Intelligence in Power-Line Communication Systems, 2009. La matriz de transmisión $T_{f}$ para una línea de transmisión se puede expresar a partir de la ecuación (6).

$$
T_{f}=\left[\begin{array}{cc}
A & B \\
C & D
\end{array}\right]=\left[\begin{array}{cc}
\cosh \gamma l & Z_{C} \operatorname{senh} \gamma l \\
\frac{1}{Z_{C}} \operatorname{senh} \gamma l & \cosh \gamma l
\end{array}\right]
$$

En la ecuación (6) $\gamma$ es la constante de propagación, $Z_{c}$ es la impedancia característica de la línea y $l$ es la longitud de la línea. Es posible calcular la constante de propagación y la impedancia característica usando los parámetros eléctricos encontrados por Bostoen y Van de Weil.

Resulta útil describir la red de admitancia $Y$ de dos puertos en términos de los parámetros de transmisión de $T_{f}$ mediante la ecuación (7) (Dorf y Svoboba, 2006).

$$
\left[\begin{array}{l}
I_{1} \\
I_{2}
\end{array}\right]=Y\left[\begin{array}{l}
V_{1} \\
V_{2}
\end{array}\right]=\left[\begin{array}{cc}
\frac{A}{B} & -\frac{1}{B} \\
-\frac{1}{B} & \frac{A}{B}
\end{array}\right]\left[\begin{array}{l}
V_{1} \\
V_{2}
\end{array}\right]
$$

Considerando que un hilo está aterrizado para todas las líneas de transmisión de dos hilos que 
componen la red, entonces solo se considera un término de la ecuación (7) para cada línea de transmisión, el cual se muestra en la ecuación (8).

$$
I_{1}=\frac{A}{B} V_{1}-\frac{1}{B} V_{2}
$$

En la ecuación (8) $I_{1}$ y $V_{1}$ son la corriente y la tensión a la entrada a una línea de transmisión que está conectada a un nodo con tensión $V_{1} \cdot V_{2}$ es el voltaje en el otro extremo de la línea, la cual puede estar conectada a una carga final u otra línea de transmisión. De acuerdo con la ley de corrientes de Kirchhoff, la suma de corrientes en un nodo debe ser cero. Si en un nodo $k$ están conectadas $N$ líneas de transmisión, las cuales a su vez están conectadas a otros $N$ nodos, la sumatoria de corrientes en el nodo $k$, aplicando la ecuación (8), es la que se describe mediante la ecuación (9).

$$
0=V_{k}^{0} \sum_{i=1}^{N} \frac{A_{i}}{B_{i}}-\sum_{i=1}^{N} \frac{V_{k}^{i}}{B_{i}}
$$

En la ecuación (9) $V_{k}^{0}$ es la tensión en el nodo $k$, $V_{k}^{i}$ son las tensiones de los $N$ nodos adyacentes al nodo $k$ conectados por las $N$ líneas de trans- misión conectadas al nodo $k ; B_{i}, \mathrm{y} A_{i}, B_{i}$ son los elementos de la matriz de transmisión de la línea que conecta el nodo $k$ con el nodo $i$. En la ecuación (9) se considera que todas las corrientes son salientes desde el nodo $k$. Para incluir los ramales terminados en una carga, se puede demostrar que hay que modificar la ecuación (9) para incluir a $Y^{v}$, donde $Y^{v}$ es la admitancia de entrada del ramal (ecuación (10)).

$$
0=V_{k}^{0} \sum_{i=1}^{N}\left(\frac{A_{i}}{B_{i}}+Y_{k}^{v}\right)-\sum_{i=1}^{N} \frac{V_{k}^{i}}{B_{i}}
$$

Al aplicar adecuadamente la ecuación (9) a cada línea de transmisión de la red residencial es posible desarrollar una herramienta que calcule las tensiones en cada nodo y carga de la red, lo que habilita el cálculo de $|\mathrm{H}(\mathrm{f})|$ entre puntos de la red, como efectivamente se hizo. Esta herramienta fue validada con resultados obtenidos por Bostoen y Van de Weil (2003). En la figura 3 se observa el caso de $|\mathrm{H}(\mathrm{f})|$ en una red con un nodo y en la figura 4 el caso de $|\mathrm{H}(\mathrm{f})|$ en una red con seis nodos. La herramienta despliega los resultados desde 1,8 $\mathrm{MHz}$ hasta $30 \mathrm{MHz}$.
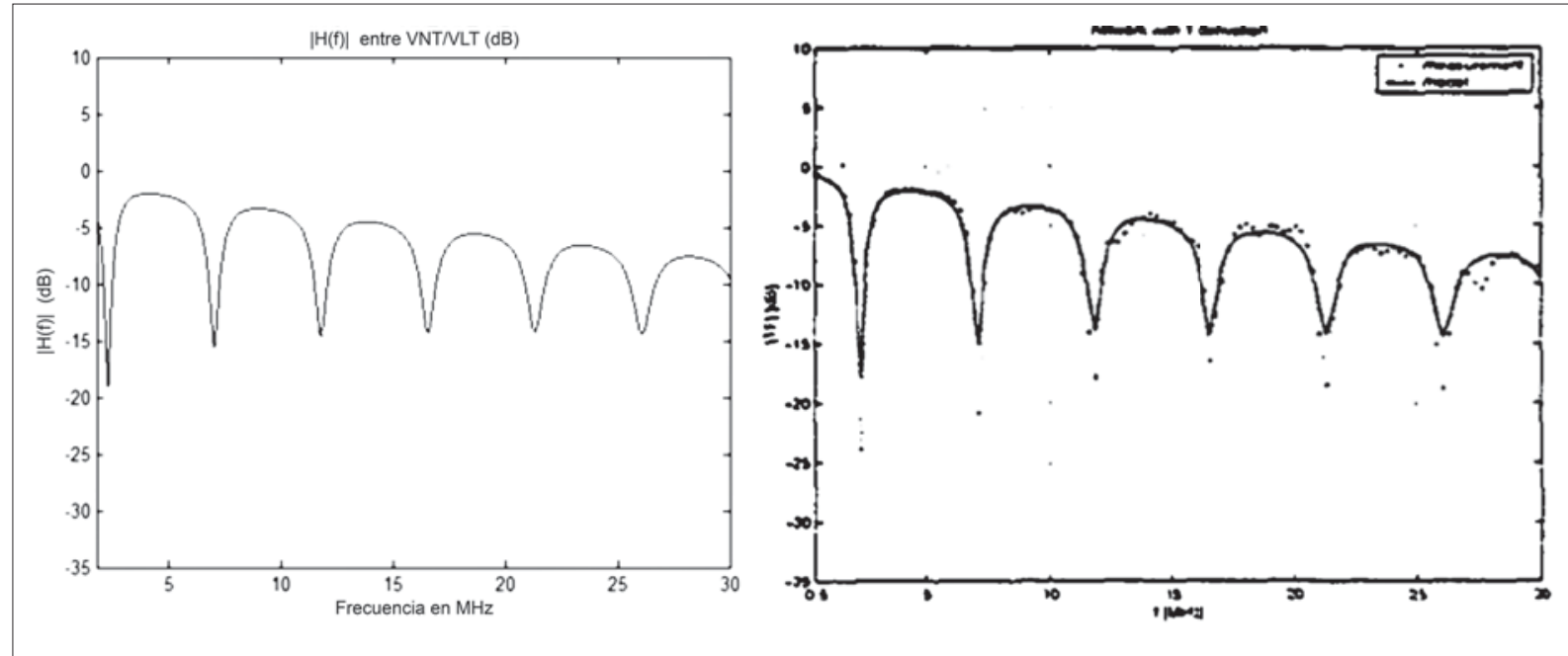

Figura 3. $|H(f)|$ en una red de 1 nodo. Izquierda, resultado de la herramienta desarrollada Fuente: Bostoen y Van de Weil (2003). 


\section{investigación}

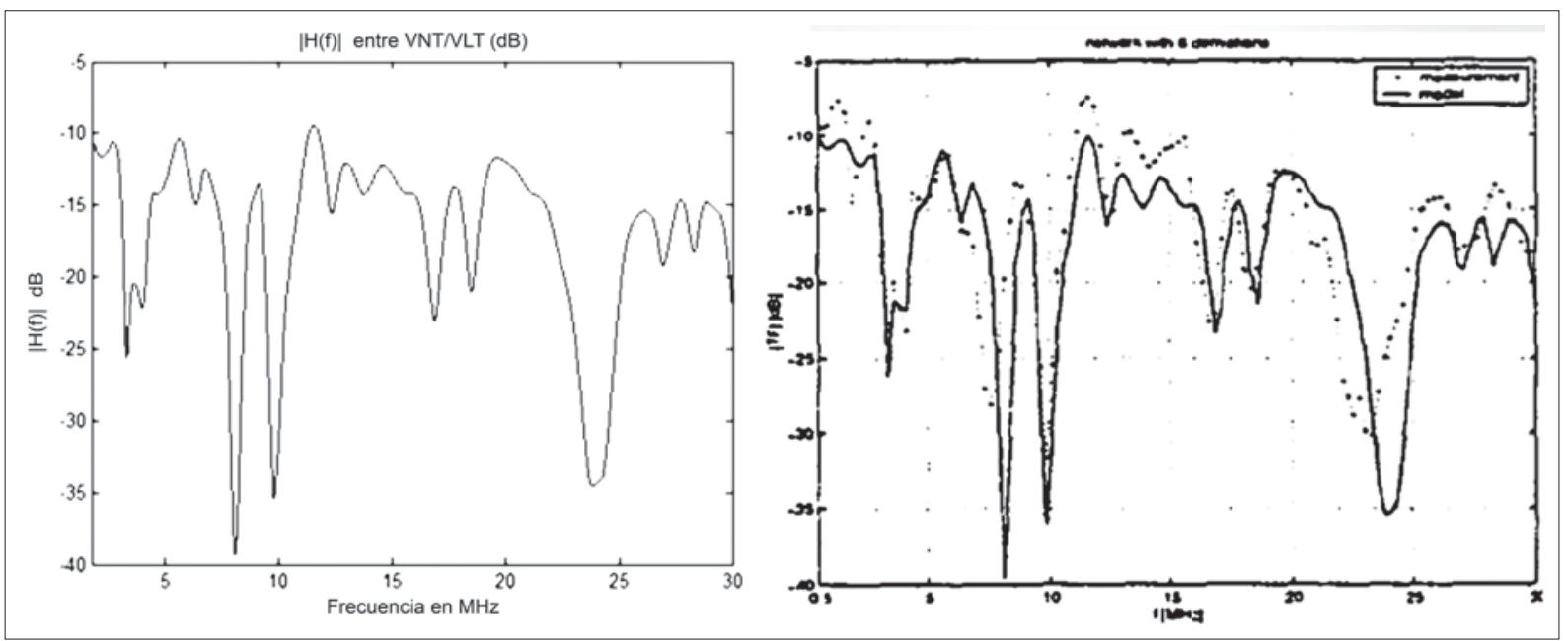

Figura 4. $|\mathrm{H}(\mathrm{f})|$ en una red de 6 nodos. Izquierda, resultado de herramienta desarrollada

Fuente: Bostoen y Van de Weil (2003).

Tanto en la figura 3 como en la figura 4 se observa coincidencia en los resultados. Sin embargo, el modelo de redes de dos puertos, aunque permite obtener las magnitudes de la función de transferencia entre puntos de la red, no deja vislumbrar mecanismos propios de las redes residenciales. Si dichos mecanismos son encontrados, estos potenciarían algún posible control sobre la interferencia. En el siguiente apartado se explora este aspecto.

\section{Uso del método sintético}

Este método de modelación (también conocido como top-down) opera desde un punto meramente fenomenológico, observándose el comportamiento del canal como un todo. Con este método Zimmermann y Dostert (1999, 2002), lograron reconocimiento con la formulación de su modelo llamado multicamino y su posterior comprobación práctica en una red de acceso de un nodo. Este método es similar a la técnica de diagrama de celosía de Bewley (Bewley, 1931) y puede expresar la función de transferencia del canal mediante la ecuación (11).

$$
H(f)=\sum_{i=1}^{N^{\prime}} g_{i} \cdot e^{-\left(a_{0}+a_{1} f^{k}\right) d_{i}} \cdot e^{-j 2 \pi f \frac{d_{i}}{v_{p}}}
$$

En la ecuación (11) los valores de $g_{i}$ (los cuales pueden ser positivos o negativos $y$, aunque existen expresiones analíticas para ellos, estos son medidos con precisión en el laboratorio en virtud de que se sigue el método sintético) se relacionan con fenómenos de reflexión y transmisión a lo largo del camino $i$ de propagación, y siendo $d_{i}$ la longitud de dicho camino, $v_{p}$ la velocidad de fase, $a_{0} \mathrm{y} a_{1}$ constantes de los cables conductores, y $k$ un valor entre cero y 1 . El término de atenuación se relaciona con las pérdidas de señal RF a lo largo de la línea y son el resultado del efecto piel y de las pérdidas en el dieléctrico. El término de retardo es la porción de constante de fase. Sin embargo, este modelo tiene dos limitaciones importantes:

- No se sabe la cantidad de caminos que se deben incluir y solo se encuentra cuando se compara el resultado del modelo con las mediciones hechas sobre el canal físico. En ese momento se habrán encontrado los $\mathrm{N}^{\prime}$ caminos necesarios para que el modelo funcione.

- Se deben hacer mediciones sobre el canal, que pueden ser previas, las cuales solo serán válidas para el canal específico.

Estas limitaciones llevan a que sean muy reducidas las posibilidades de poder hacer prediccio- 
nes del comportamiento de redes y canales. Sin embargo, es posible pensar que como modelo no solo debe ser aplicable a las redes de acceso, sino también a las redes residenciales.

\section{Escenario típico residencial}

Como caso de análisis se presenta en la figura 5 la red residencial propuesta por Tucci et al, 2007. En este caso las consideraciones se centran entre los puntos TX y RX. El interruptor L coloca sobre el ramal 6 una carga igual a la impedancia característica de la línea o termina la línea en circuito abierto. Las cargas FAN, COFFEE MACHINE y TV son cargas no lineales, las cuales son modeladas siguiendo la técnica de Bardama et al. (2009).

\section{APORTES TEÓRICOS PARA HALLAR UN MÉTODO DE MODIFICACIÓN DE |H(F)|}

En este apartado se consignan los aportes teóricos que permitieron desarrollar un algoritmo que de manera sistemática permite la modificación de $|\mathrm{H}(\mathrm{f})|$.



Figura 5. Red eléctrica residencial típica con longitudes (números pequeños) y numeración de ramales (números grandes)

Fuente: Tucci et al. (2007). Blind Channel Estimation for Power-line Communications by a Kohonen Neural Network.

\section{Uso del método hipotético-deductivo: ganancia por distancia}

Para explicar analíticamente el comportamiento de los canales, se asumió (hipótesis) que la base teórica del modelo top-down para canales de acceso de alguna forma también podría explicar fenómenos de las redes residenciales con más de un nodo. De hecho, la red de topología de bus puede ser construida interconectando redes de un nodo. La ecuación (11) se puede escribir como la ecuación (12).

$$
\begin{gathered}
H(f)=\sum_{i=1}^{N^{\prime}}\left(g_{i} e^{-a(f) d_{i}} \cos w_{i}\right)+ \\
j \sum_{i=1}^{N^{\prime}}\left(-g_{i} e^{-a(f) d_{i}} \operatorname{sen} w_{i}\right)
\end{gathered}
$$

donde, $a(f)=a_{0}+a_{1} f^{k}, w_{i}=2 \pi f \tau_{i}, \tau_{i}=d_{i} / v_{p}$. De la ecuación (12) se puede derivar $|H(f)|^{2}$ (ecuación (13)).

$$
\begin{gathered}
|H(f)|^{2}=\left(\sum_{i=1}^{N^{\prime}} g_{i} e^{-a(f) d_{i}} \cos w_{i}\right)^{2}+ \\
\left(\sum_{i=1}^{N^{N}} g_{i} e^{-a(f) d_{i}} \operatorname{sen} w_{i}\right)^{2}
\end{gathered}
$$

Desarrollando la ecuación (13) para $\mathrm{N}^{\prime}=1$ se obtiene la ecuación (14).

$$
\left(|H(f)|^{2}\right)_{1}=g_{1}^{2} e^{-2 a(f) d_{1}}
$$

Desarrollando la ecuación (13) para $\mathrm{N}^{\prime}=2$ se obtiene la ecuación (15).

$$
\begin{gathered}
\left(|H(f)|^{2}\right)_{2}=\left(|H(f)|^{2}\right)_{1}+ \\
g_{1}^{2} e^{-2 a(f) d_{2}}\left[1+2\left(g_{1} / g_{2}\right) e^{-2 a(f) d_{1}} \cos \left(w_{1}-w_{2}\right)\right]
\end{gathered}
$$

Desarrollando la ecuación (13) para $\mathrm{N}^{\prime}=3$ se obtiene la ecuación (16).

$$
\begin{gathered}
\left(|H(f)|^{2}\right)_{3}=\left(|H(f)|^{2}\right)_{2}+ \\
g_{3}^{2} e^{-2 a(f) d_{3}}\left[1+2 \sum_{i=1}^{2} \frac{g_{i}}{g_{3}} e^{-2 a(f) d_{i}} \cos \left(w_{1}-w_{3}\right)\right]
\end{gathered}
$$




\section{investigación}

Recordando que en una línea de transmisión $v_{p}=\lambda * \mathrm{f} \mathrm{m} / \mathrm{s}$, se puede generalizar $\mathrm{N}^{\prime}>1$ para (ecuación (17))

$$
\left(|\mathrm{H}(\mathrm{f})|^{2}\right)_{\mathrm{N}^{\prime}}=\left(|\mathrm{H}(\mathrm{f})|^{2}\right)_{\mathrm{N}^{\prime}-1}+\Delta_{\mathrm{N}^{\prime}}
$$

siendo,

$$
\begin{gathered}
\Delta_{N^{\prime}}=g_{N^{N}}^{2} e^{-2 a(f) d_{N^{\prime}}}\{1+ \\
\left.2 \sum_{i=1}^{N^{\prime-1}} g_{i} g_{N^{\prime}} e^{-2 a(f) d_{i}} \cos \left[2 \pi \cdot \frac{d_{i}-d_{N^{\prime}}}{\lambda}\right]\right\}
\end{gathered}
$$

La función $\Delta_{N^{\prime}}$ (ecuación (18) describe la influencia en la magnitud de la función de transferencia al cuadrado, al considerarse un camino adicional al número de caminos $\mathrm{N}^{\prime}-1$, observándose de inmediato el comportamiento discreto de $\left(|\mathrm{H}(\mathrm{f})|^{2}\right)_{\mathrm{N}}$. Además se observa claramente en la ecuación (18) que el factor $\Delta_{N \mathrm{c}}$ es dependiente de la diferencia de longitud de caminos (lo que indica dependencia de la configuración geométrica de la red). Si se define $G d_{i}$ a partir de la ecuación (19).

$$
G d_{i}=\cos \left[2 \pi \cdot \frac{\left|d_{i}-d_{N^{N}}\right|}{\lambda}\right]
$$

y llamando a $G d_{i}$ ganancia por distancia, se observa que esta ganancia puede tener un valor positivo, negativo o cero dependiendo del valor $\left|d_{i}-d_{N^{\prime}}\right|=k \cdot \lambda$ con $k \in \mathbb{R}^{+}$, como se muestra en la Figura 6.

Al observar la ecuación (18) interesarán los casos donde $g_{i} / g_{N^{n}}$ y $G d_{i}$ tengan el mismo signo, ya que esto significa un valor positivo en la sumatoria y la suma de muchos valores positivos podría llevar a que $\Delta_{N^{\prime}}$ sea mayor a $0 \mathrm{~dB}$. En la figura 7 se muestra un caso en el cual $\left|d_{i}-d_{N^{\prime}}\right|\left(G d_{i}\right.$ máximo).

En los cálculos de las longitudes de los caminos de propagación se ha tenido en cuenta que en las líneas de transmisión terminadas en circui-

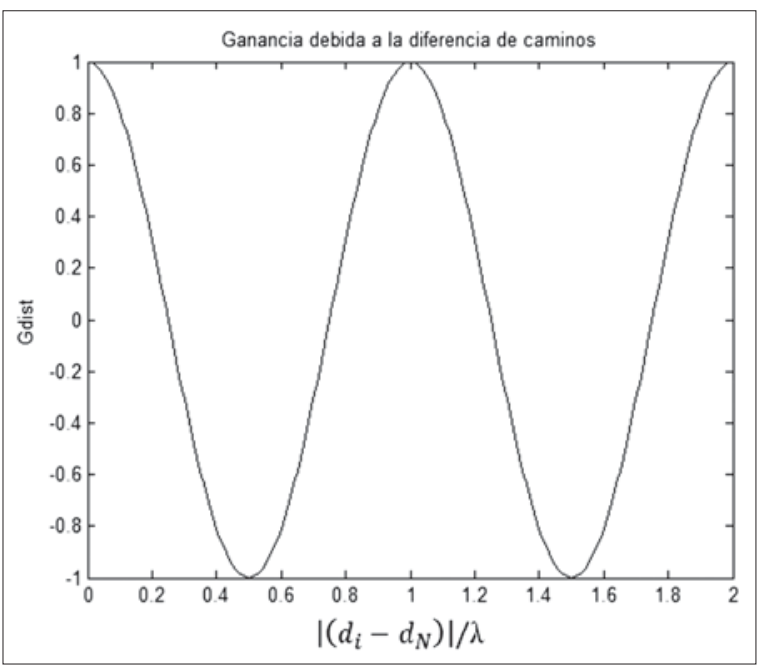

Figura 6. Ganancia por distancia debida a la diferencia de caminos de propagación

to abierto la onda de tensión incidente se refleja exactamente, de la misma manera como si fuera a continuar a lo largo de una línea infinitamente larga. El camino de propagación del primer y segundo recorrido sería la adición de las siguientes distancias, donde las cantidades entre paréntesis muestran caminos creados por reflexión (ecuación (20) y (21)).

Distancia 1 er. camino $=$

$3+1+11+1+13+(13+1+11+3+3)=60 \mathrm{~m}$

Distancia 2 do. camino $=$

$$
\begin{gathered}
3+1+3+4+3+1+[(1+3+3) \cdot 5]+ \\
(3+4+3) 3+4+3=60 \mathrm{~m}
\end{gathered}
$$

Si se considera que la velocidad de fase está alrededor de $188280000 \mathrm{~m} / \mathrm{s}$ (estimada computacionalmente) y si además se asume que en este caso $\lambda=60 \mathrm{~m}$, entonces se podría presumir que se presentaría una posible ganancia de $|\mathrm{H}(\mathrm{f})|$ en una frecuencia determinada a partir de la ecuación (22).

$$
f=\frac{v_{p}}{\lambda}=\frac{188280000}{60}=3,1138 \mathrm{MHz}
$$

En la figura 8 se muestra $|\mathrm{H}(\mathrm{f})|$ entre TX y la carga del ramal 21 calculada con la herramienta 


\section{investigación}



Figura 7. Dos posibles caminos de propagación de 60 metros en la red residencial

Fuente: Tucci et al. (2007). Blind Channel Estimation for Power-line Communications by a Kohonen Neural Network.

bottom-up. Se observa efectivamente que existe en $3,138 \mathrm{MHz}$ una ganancia que está muy cercana al pico máximo de $|\mathrm{H}(\mathrm{f})|$.

Una $|\mathrm{H}(\mathrm{f})|$ como la mostrada en la figura 8 no es común, pero se puede explicar con la teoría de $G d_{i}$. Del cálculo realizado en las ecuación (21) y (22) se puede concluir que las longitudes de los ramales y sus cargas influyen en $|\mathrm{H}(\mathrm{f})|$, al punto de poder producir ganancias.

\section{Construcción discreta de la capacidad de canal en redes residenciales}

Si se considera que la técnica de transmisión adoptada para sistemas BPL hogareña es OFDM (con $\mathrm{N}$ portadoras) y que la densidad espectral de potencia del ruido en redes residenciales varía sustancialmente, para intervalos lineales del canal se podrá expresar la capacidad a partir de la ecuación (23).

$C=\Delta f \cdot \sum_{i=1}^{N} \log _{2}\left[1+\frac{S_{t}\left(f_{i}\right) \cdot\left|H\left(f_{i}\right)\right|^{2}}{S_{n}\left(f_{i}\right)}\right]\left(\frac{\text { bits }}{s}\right)$
En este estudio se asumen $\Delta f=9 \mathrm{KMz}$ especificada por la FCC (Federal Communications Commission, 2009) para el caso de redes BPL, $S_{t}\left(f_{i}\right)$ la DEP de transmisión definida por IEEE 1901, $S_{n}\left(f_{i}\right)$ la DEP de ruido típica residencial encontrada por Benyoucef (Benyoucef, 2003). Aumentos en $\left|H\left(f_{i}\right)\right|^{2}$ se reflejarán en aumento de C.



Figura 8. $|\mathrm{H}(\mathrm{f})|$ entre TX y la carga del ramal 21 (con $\mathrm{L}$ abierto) con frecuencia para máximo $\mathrm{Gd}_{i}$ ( $\left.\left|d_{i}-d_{N^{\prime}}\right|=0\right)$ 


\section{investigación}

Sería posible usar la ecuación (17) en la ecuación (23), obteniendo la ecuación (24).

$C_{N^{N}}=\Delta f \cdot \sum_{i=1}^{N} \log _{2}\left[1+\frac{S_{t}\left(f_{i}\right) \cdot\left|H\left(f_{i}\right)\right|_{N^{N}}^{2}}{S_{n}\left(f_{i}\right)}\right]\left(\frac{\text { bits }}{s}\right)$

La ecuación (24) define la capacidad para redes BPL residenciales, la cual demuestra un comportamiento discreto al depender de $\left(\left|H\left(f_{i}\right)\right|^{2}\right)_{N}$ y es dependiente de la longitud de los caminos de propagación (incluidas las longitudes de los ramales) y de las cargas de terminación en los ramales. Entre más $N^{\prime}$ caminos de propagación se habiliten, la sumatoria de $\Delta_{N^{\prime}}$ tendrá mayor oportunidad de agregar términos positivos. La habilitación de $N^{\prime}$ caminos puede hacerse colocando sobre los ramales cargas que reflejen la onda (p.e. $\mathrm{ZL}=100 \mathrm{Zc}$ ). Cuando no se logre aumentar la sumatoria de $\Delta_{N^{\prime}}$ operando con un ramal, se coloca una carga acoplada $(\mathrm{ZL}=\mathrm{Zc})$.

\section{Direccionalidad en el nodo transmisor}

Ahora se asumirá que el transmisor $\left(V_{S}\right)$ está situado en el ramal de la red de un nodo, como se muestra en la figura 9. En este caso se definen $l_{1} B=\lambda / 4, r_{21}=l_{2 B} / l_{1 B}$ y $r_{31}=l_{3 B} / l_{1 B}$. El estudio se hace con los parámetros eléctricos de la línea $4 \times 95 \mathrm{~mm}^{2} 1-(2+3+4)$ para todos los ramales y las impedancias en los puntos 2 y 3 son 100 veces la impedancia característica de las líneas 2-B y 3-B respectivamente.

Considerando las distancias de la red residencial, interesará acotar los valores de $r_{31}$ y $r_{21}$ a valores entre 1 y 2 , ya que por ejemplo si se considera que el ramal tiene una longitud $\lambda / 4 @ 20,1 \mathrm{MHz}$, se estarían considerando longitudes de alrededor de $2,34 \mathrm{~m}$.

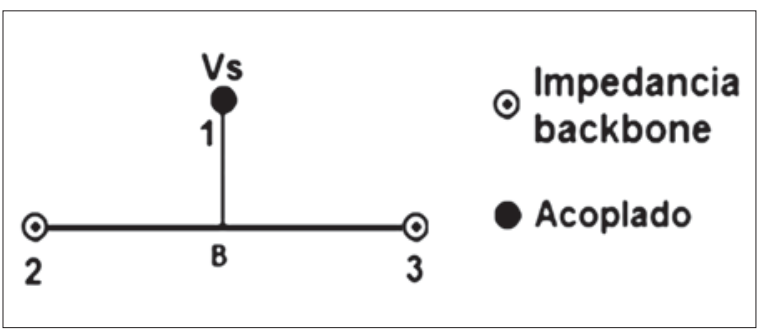

Figura 9. Red de un nodo con el transmisor situado en el ramal 1-B (Punto 1)

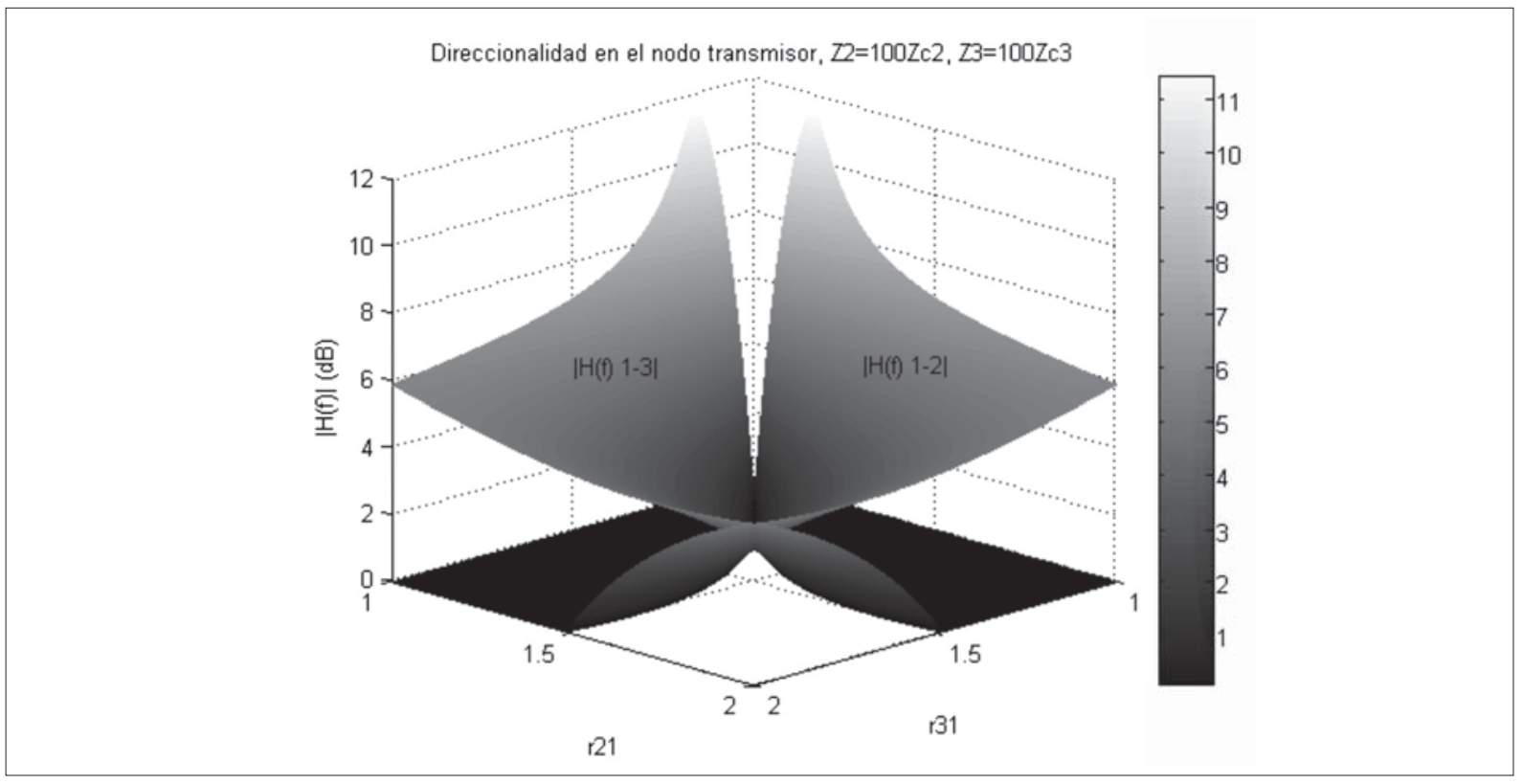

Figura 10. Superposición de $|\mathrm{H}(\mathrm{f}) 1-2|$ con $|\mathrm{H}(\mathrm{f}) 1-3|$ cuando se transmite por el ramal 1-B@ $20,1 \mathrm{MHz}$ 


\section{investigación}

La figura 10 es obtenida mediante el uso de la herramienta bottom-up y muestra la superposición de las $|\mathrm{H}(\mathrm{f})|$ 's cuando se sale por los ramales 2 y 3 de figura 9, para $|H(f)|>0 d B$. Se observa que existen puntos donde para $|\mathrm{H}(\mathrm{f}) 1-3|>0$ corresponde $|H(f) 1-2| \leq 0$ y viceversa, es decir se produce una orientación de la transmisión.

La unidireccionalidad se puede obtener ajustando la longitud del ramal TX (1-B). De forma análoga se puede demostrar que se produce ganancia en RX ajustando la longitud del ramal RX.

\section{ALGORITMO DE VARIACIÓN DE LONGITUD DE RAMAL (AVLR)}

Con los anteriores aportes teóricos se construyó el algoritmo AVLR (figura 11) que permite disminuir valores de $|\mathrm{H}(\mathrm{f})|$ y así obtener mayor reducción de la DEP en TX usando ATPM.

Las convenciones adoptadas para el diagrama de flujo de la figura 11 son:

$\mathrm{N}=$ número de ramales participantes

$\mathrm{i} \leq \mathrm{N}-2=$ índice referente a ramales diferentes a ramal RX (receptor) o ramal TX (transmisor).

$\mathrm{i}=\mathrm{N}-1=$ índice referente al ramal receptor.

$\mathrm{i}=\mathrm{N}=$ índice referente al ramal transmisor.

Zci $=$ impedancia característica del ramal i.

Zc_N-1 = impedancia característica del ramal receptor.

Zc_N = impedancia característica del ramal transmisor.

$\mathrm{ZN}-1$ = impedancia de carga del ramal receptor.

$\mathrm{ZN}=$ impedancia de carga del ramal transmisor.
$\mathrm{Zi}=$ impedancia de carga del ramal i (puede tomar valores de Zció 100Zci para ramales diferentes a TX y RX, ZN =Zc_Npara ramal TX (acoplado), $\mathrm{ZN}-1=5 \mathrm{Zc} \_\mathrm{N}-1$ para ramal $\mathrm{RX}$ ).

Cmax $=$ Capacidad máxima entre los puntos TX y RX.

$\mathrm{Ci}=\mathrm{Cmax}$ al procesar el ramal $\mathrm{i}$.

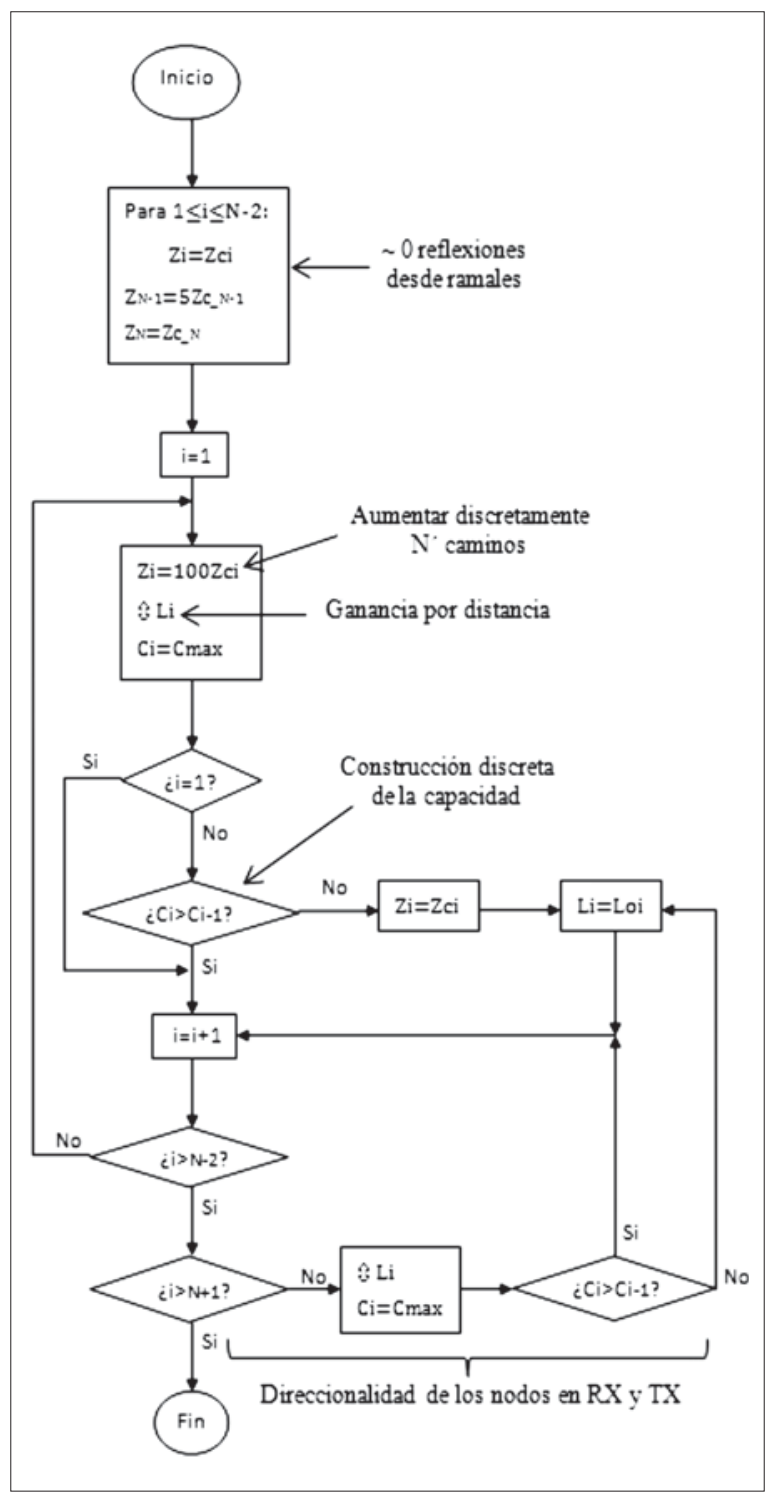

Figura 11. Diagrama de flujo del algoritmo AVLR Fuente: elaboración propia. 
$\mathrm{Li}=$ longitud del ramal $\mathrm{i}$.

Loi $=$ longitud original del ramal $\mathrm{i}$.

全 $\mathrm{Li}=$ variar $\mathrm{Li}$.

La tabla 1 muestra los pasos al usar el algoritmo AVLR con el canal TX-RX de la figura 5.

En la figura 12 se observa que la mínima $|\mathrm{H}(\mathrm{f})|$ en el canal original es $-15,3174 \mathrm{~dB}$ y cuando se aplica el algoritmo AVLR es -3,6141 dB. Por tanto, desde la perspectiva de ATPM, con el canal original se podría disminuir la DEP de transmisión 30$15,3174=14,6826 \mathrm{~dB}$ y con el canal modificado $30-3,6141=26,3859 \mathrm{~dB}$.

\section{CONCLUSIONES}

Como se ha demostrado, es posible mejorar el desempeño de la técnica ATPM usando tres aportes teóricos hallados en esta investigación: ganancia por distancia, construcción discreta de la capaci- dad de canal y direccionalidad en los nodos de transmisión y recepción. La mejora obtenida en la DEP de transmisión usando ATPM + AVLR en el caso de estudio fue de 26,3859 dB. Este resultado hace que el rendimiento de la técnica ATPM + AVLR sea comparable a la de ranuras dinámicas $(30 \mathrm{~dB})$ y mejor que las demás técnicas mencionadas en el estado del arte. Esta mejora hace factible la reducción recomendada por ITU-R de $48 \mathrm{~dB}$, ya que mediante la combinación ATPM + AVLR y de ranuras dinámicas se lograría una reducción teórica de $30 \mathrm{~dB}+26,4 \mathrm{~dB}=56,4 \mathrm{~dB}$. Adicionalmente, la técnica ATPM+AVLR como técnica individual logra el cumplimiento de lo especificado por la FCC, ya que se lograría transmitir con $-55 \mathrm{dBm} / \mathrm{Hz}-26,4 \mathrm{~dB}=-81.4 \mathrm{dBm} / \mathrm{Hz}$, recordando que lo necesario sería $-72 \mathrm{dBm} / \mathrm{Hz}$. Desde el punto de vista teórico, se demuestra que el canal residencial puede tener un tratamiento determinístico y su respuesta puede ser alterada, diferenciándolo así de los modelos modernos de canales como el inalámbrico.

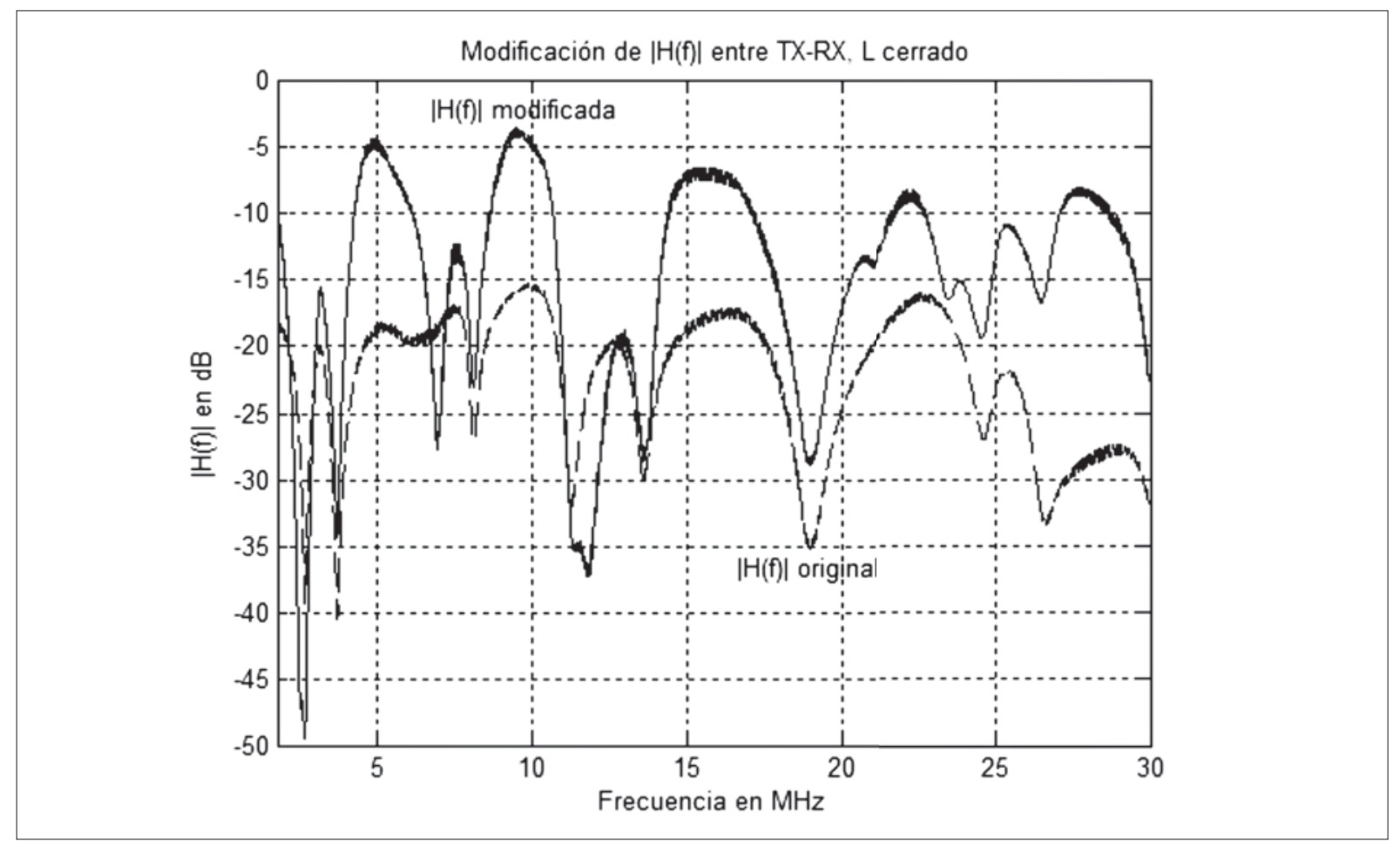

Figura 12. Resultado al aplicar el algoritmo AVLR al canal TX-RX de la figura 5 con $L$ cerrado 


\section{investigación}

Tabla 1. Evolución del algoritmo AVLR para el canal TX-RX de la figura 5. Cálculo con DEP de transmisión = $-52 \mathrm{dBm} / \mathrm{Hz}$

\begin{tabular}{|c|c|c|c|c|}
\hline Paso & Número de ramal & Longitud final $(\mathrm{m})$ & Factor k de kZc en la carga & Capacidad TX-RX (Mbps) \\
\hline 1 & 25 & 7,5 & 100 & 570,38 \\
\hline 2 & 21 & 1,6 & 100 & 573,52 \\
\hline 3 & 20 & 6,9 & 100 & 573,73 \\
\hline 4 & 19 & 6,9 & 100 & 573,91 \\
\hline 5 & 15 & 1,8 & 100 & 577,64 \\
\hline 6 & 13 & 2,7 & 5 & 592,75 \\
\hline 7 & 8 & 0,5 & 100 & 606,72 \\
\hline 8 & 1 & 0,1 & 1 & 608,16 \\
\hline
\end{tabular}

\section{REFERENCIAS}

Babic, M. et al. (2005). Theoretical Postulation of PLC Channel Model. Technical Report, Open PLC European Research.

Barmada, S. et al. (2009). Response Bounds of Indoor Power-Line Communication Systems With Cyclostationary Loads. IEEE Transactions on Power Delivery, 24 (2), 596-603.

Benyoucef, D. (2003). A New Statistical Model of the Noise Power Density Spectrum for Powerline Communication. International Symposium on Power-Line Communications and Its Applications, pp. 136-141. Kyoto.

Bewley, V. (1931, june). Traveling Waves on Transmission Systems. Transactions of the American Institute of Electrical Engineers, 532-550.

Bostoen, T., Van de Weil, O. (2003). Modeling the low-voltage power distribution network in the frequency band from $0.5 \mathrm{Mhz}$ to 30 Mhz for broadband powerline communications. Proceedings of Seminar on Broadband
Communications, Accessing, Transmission, Networking, pp. 171-178. Zurich.

Chaaban, M., et al. (2009). Reduction of Power Field Radiation for PLC Applications. ACTEA'09, Advances in Computational Tools for Engineering Applications, pp. 422-426. Zouk Mosheh.

Cheng, W. (2003). Home Networking Basis (1a. ed.). New Jersey: Prentice Hall.

Dorf, R. y Svoboba, J. (2006). Circuitos Eléctri$\cos$ (6 ed.). México D.F., México: Alfaomega.

Favre, P. et al. (2009). Radiation and disturbance mitigation in PLC networks. 20th Int. Zurich Symposium on EMC, 5-8.

Federal Communications Commission. (2009). § 15.209 Radiated Emission Limits. 810.

IEEE Communications Society. (2010). IEEE Standard for Broadband over Power Line Networks: Medium Access Control and Physical Layer Specifications, IEEE 


\section{investigación}

Std 1901-2010. New York: IEEE STANDARDS ASSOCIATION.

ITU-R SM Series. (2011). Impact of power line telecommunication systems on radiocommunication systems operating in the LF, $\mathrm{MF}, \mathrm{HF}$ and VHF bands below $80 \mathrm{MHz}$.

Mekuria, G. (2008). Mitigating EMI Powerline Communications Using Carrier-less UWB Pulses. Disertación para obtener el titulo de Doctor en Ingeniería, Universidad de Duisburg-Essen, Duisburg-Essen.

Pang, S. et al. (2010). Feasibility Study of a New Injection Method for EMI Reduction in Indoor Broadband PLC Networks. IEEE Transactions on Power Delivery, 25 (4), 2392-2398.

Schwager, A. (2010). Powerline Communications: Significant Technologies to become Ready for Integration. Disertación para obtener el título de Doctor en Ingeniería, Universidad Duisburg-Essen, DuisburgEssen.

Stott, J. (2006, julio). Potencials threats to radio services from PLT systems. EBU Technical Review, 1-15.

Tucci, M. (2009). Computational Intelligence in Power-Line Communication Systems. Beau Bassin, Mauritius: VDM Publishing House.
Tucci, M. et al. (2007). Blind Channel Estimation for Power-line Communications by a Kohonen Neural Network. IEEE International Symposium on Power-line Communications and Its Applications, pp. 35-40. Pisa.

Vukicevic, A. et al. (2007). On the Impact of Mitigating Radiated Emissions on the Capacity of PLC Systems. IEEE International Symposium on Power Line Communications and Its Applications, ISPLC '07, pp. 487492. Udine.

Weling, N. (2011). Feasibility Study on Detecting Short Wave Radio Stations on the Powerlines for Dynamic PSD Reduction as Method for Cognitive PLC. IEEE International Symposium on Power Line Communications and Its Applications, pp. 311-316.

Zimmermann, M. y Doster, K. (1999). A MultiPath Signal Propagation Model for the Power Line Channel in the High Ferquency Range. IEEE International Symposium on Power Line Communications and Its Applications, 45-51.

Zimmermann, M. y Dostert, K. (2002). A Multipath Model for the Powerline Channel. IEEE Transactions on Communications. 